\title{
Estudo descritivo de quedas entre menores de 15 anos no município de Londrina (PR, Brasil)
}

\author{
Descriptive study of fall among children under the age of 15 \\ in the municipality of Londrina (Paraná, Brazil)
}

Christine Baccarat deGodoy M artins ${ }^{1}$

Selma $M$ affei de Andrade ${ }^{2}$

${ }^{1}$ Faculdade deEnfermagem, UniversidadeFederal de $M$ ato Grosso. Rua Fortaleza 70 , Jardim Paulista. 78065-350 Cuiabá MT. leocris2001@terra.com.br ${ }^{2}$ Departamento deSaúce Coletiva, U niversidade Estadual deLondrina.
Abstract This study aims at analyzing, from the epidemiological point of view, occurrences of falls among children under the age of 15 who live in Londrina, Paraná, and were treated at emergency departments, hospitalized or passed away due to such event in 2001. Data were obtained from registries of general hospitals and from the $\mathrm{M}$ ortality Information Nucleus. 2,991 children victims of falls were analyzed, representing an incidence rate of 25.3 per 1000 children. N on-fatal hospitalization ratewas $3.9 \%$; one death occurred in an emergency department. The risk of suffering falls was higher among boys, except with children aged less than one year. M ost frequently injured by falls were the groups of one year old (coefficient of 46.0 per 1000 children) and of two years old (coefficient of 38.4 per 1000 children). The most affected body region was head/neck (55.2\%) and superficial traumatism wasthemost frequent kind of injury (46.4\%). Falls occurred mainly from bed/cradle, furniture/couch, tricycle/skates, staircase/degrees, playground equipment, wall/gate/roof/balcony, hammock, tree and stroller. Findings show a high falls incidence among children and adolescents and contribute to increasing epidemiological knowledge of such events and so grounding the planning of preventive and control measures.

Key words Falls, Child, Domestic accidents
Resumo 0 objetivo deste estudo foi analisar, do ponto de vista epidemiológico, a ocorrência das quedas entre menores de 15 anos residentes em Londrina, Paraná, queforam atendidos em serviços de emergência e internação ou que morreram devido a essas causas em 2001. Os dados foram obtidos em prontuários dos hospitais gerais e no $\mathrm{N}$ úcleo de Informação em M ortalidade. Foram estudadas 2.991 crianças víti mas de queda, representando taxa deincidência de 25,3 por 1.000 crianças. A taxa de internação não fatal foi de $3,9 \%$, ocorrendo um óbito no pronto-socorro. 0 risco de sofrer queda foi maior no sexo masculino, exceto nos menores de um ano. As faixas etárias mais atingidas foram de 1 ano (coeficientede 46,0 por 1.000 crianças) e 2 anos (coeficiente de 38,4 por 1.000 crianças). Asregiões corpóreas mais afetadas foram a cabeça e o pescoço $(55,2 \%)$, e 0 traumatismo superficial foi o tipo de lesão mais frequente $(46,4 \%)$. As quedas ocorreram, principalmente, deleito/berço/cama, mobília/sofá, motoca/velocípede/patins/skate, escada/degrau, equipamento de playground, muro/portão/tel hado/varanda, rede, árvore e carrinho de bebê. Os achados permitem concluir a alta incidência de quedas entre crianças e adolescentes, contribuindo para ampliar o conhecimento epidemiológico sobre tais eventos, fornecendo subsídios para o planejamento de ações preventivas e de controle. Palavras-chave Quedas, Criança, Acidentes D omésticos 
Introdução

Atualmente, em todo o mundo, a alta incidência dos acidentes na infância, bem como a importância do seu controlee prevenção, continua despertando o interesse por estudos que possam melhor direcionar efundamentar a implementação, a execução e a avaliação de estratégias de prevenção específicas ${ }^{1-11}$.

Entre os acidentes infantis, as quedas têm sido apontadas por vários autores como o tipo de acidente mais frequente, sendo a principal causa de atendimento hospitalar e de internação $0^{5,9,10,12}$.

Além dos custos sociais, econômicos e emocionais, as quedas são responsáveis por grande parte dos traumatismos não fatais, como por exemplo déficits neurológicos persistentes em razão detraumatismos cranioencefálicos ${ }^{13,14}$, que exercem um grande impacto a longo prazo, re percutindo nafamília ena sociedadee penalizando crianças e adolescentes em plena fase de crescimento e desenvolvimento.

Nesse contexto, torna-se essencial ampliar 0 conhecimento sobreas características epidemiológicas das quedas que ocorrem entre crianças, contribuindo para a avaliação da magnitude e características do fenômeno e subsidiando o planejamento de ações preventivas que visem à redução desse importante agravo em nossa sociedade.

0 presente estudo teve o objetivo deestudar a ocorrência das quedas entre menores de 15 anos, residentes em Londrina (PR), que foram atendidos em serviços de emergência e internação ou que morreram devido a essas causas em 2001.

\section{M etodologia}

Este estudo, transversal e descritivo acerca da morbidade hospitalar e da mortalidade por quedas entre crianças e adolescentes, foi realizado nos serviços hospitalares de emergência e internação de Londrina e no Núcleo de Informação em M ortalidade (N IM) da Prefeitura de Londrina, uma vez que o óbito poderia ter ocorrido sem que 0 atendimento houvesse sido prestado por serviços de saúde.

Os critérios de inclusão para formar a população de estudo foram: menores de 15 anos de idade, residentes no município de Londrina, vítimas de quedas ocorridas no período de 01 de janeiro a 31 de dezembro de 2001, que foram atendidos em serviços de emergência ou internação hospitalar do município ou que morreram devido a esses eventos em um prazo de até um ano após o acidente, conforme preconiza a Classificação Internacional de Doenças ${ }^{15}$.

Optou-se por incluir no estudo apenas menores de 15 anos, tendo em vista as diferentes características e circunstâncias das quedas nas idades acima.

Os dados da investigação foram coletados nas fichas de pronto-socorro e nos prontuários de internação hospitalar, utilizando-se um formulário previamente testado contendo questões fechadas. Todos os formulários foram codificados manualmente pelas pesquisadoras a fim de verificar o código do tipo de acidente e tipo de lesão segundo a Classificação Internacional de Doenças, décima revisão ${ }^{15}$.

Os dados de mortalidade foram obtidos no Núcleo de Informação em M ortalidade (NIM) de Londrina, através de uma lista fornecida com todos os óbitos de crianças menores de 15 anos residentes no município, ocorridos durante 2001 e 2002, cuja causa básica de morte fosse queda. Os óbitos ocorridos em 2002 foram levantados apenas com 0 intuito de verificar se 0 acidente não tinha ocorrido no ano de 2001, porém nenhum caso foi observado.

Nas análises estatísticas foi empregado o programa computacional Epi-Info - versão 6.0.d.16, incluindo-se os óbitos que ocorreram sem assistência com vistas a formar um único banco de dados de morbimortalidade. Uma criteriosa verificação de inconsistências foi realizada através do cruzamento e verificação de dados.

Tendo por base a população estimada na mesmafaixa etária eano, calculou-seo coeficiente deincidência.

Os resultados são apresentados em forma de gráficos e tabelas, em números absolutos e relativos. Foi solicitada, aos diretores ou responsáveis de cada instituição, a autorização por escrito para 0 acesso aos prontuários e informações. A pesquisa foi aprovada pelo Comitê de Ética em Pesquisa da Universi dade Estadual de Londrina.

\section{Resultados}

0 município de Londrina, criado em 10 dedezembro de 1934, situa-se no norte do estado do Paraná. É o segundo mais populoso do estado e o terceiro da região Sul do Brasil, com cerca de 470 mil habitantes e uma taxa de urbanização de $97,0 \%{ }^{17}$. Em 2004, o coeficiente de mortalidade infantil do município foi de 9,04 (por 1.000 nascidos vivos), e o coeficiente de mortalidade por causas externas foi de 20,21 entre os menores de 15 anos ${ }^{18}$. 
Faz-se necessário destacar que o presente estudo abrange apenas as crianças atendidas em pronto-socorro ou que foram internadas ou que morreram, tratando-se, portanto, de uma incidência mínima, referente apenas àquela parcela quechegou aos serviços de atendimento deemergência ou que morreu sem assistência.

Foram estudados 2.991 casos de quedas, 0 que representou uma taxa de incidência de 25,3 por 1.000 crianças/adolescentes.

0 coeficiente de incidência das quedas foi maior no sexo masculino praticamente em todas as idades, exceto entre os menores de um ano (Gráfico 1). 0 risco desse evento émaior nafaixa etária de um a três anos em ambos os sexos, com um coeficiente total de 46,0/1.000 crianças na idadede um ano ede $38,4 / 1.000$ crianças na idadede dois anos.

A cabeça e o pescoço responderam por $55,2 \%$ das regiões corpóreas atingidas em decorrência das quedas, seguidos pelo ombro/membros superiores $(26,2 \%)$ e pelo quadril emembros inferiores $(11,9 \%)$ (Tabela 1$)$.
O tipo de lesão mais frequente foi o trauma superficial $(46,4 \%)$, seguido pelo ferimento $(20,2 \%)$ e pela fratura ( $12,1 \%)$ (Tabela 2 ).

Observou-se grande parte dos prontuários sem a informação do local onde ocorreu a queda $(81,4 \%)$. Entre os casos em que foi possível determinar, as quedas ocorreram, principalmente, de leito/berço/cama (19,2\%), motoca/velocípede/patinete/patins/skate (14,4\%), mobílias/sofás (12,4\%), árvore (8,4\%), escada/degrau (8,3\%), equipamentos de playground $(7,0 \%)$, carrinho de bebê (5,0\%), muros/portão/telhado/varanda $(4,9 \%)$, por prática deesporte $(4,5 \%)$, rede $(2,9 \%)$ e colo $(2,5 \%)$, entre outros $(10,0 \%)$.

Convém esclarecer que, no presente estudo, excluíram-se as quedas de bicicleta, que são agrupadas nos acidentes de transporte, conforme a Classificação Internacional deDoenças (CID-10) ${ }^{15}$.

Quanto ao nível de atendimento e evolução das crianças vítimas de queda, 2.872 casos $(96,0 \%)$ foram atendidos exclusivamente no pronto-socorro, 118 casos (3,9\%) necessitaram de internação (não fatal) e houve um óbito no

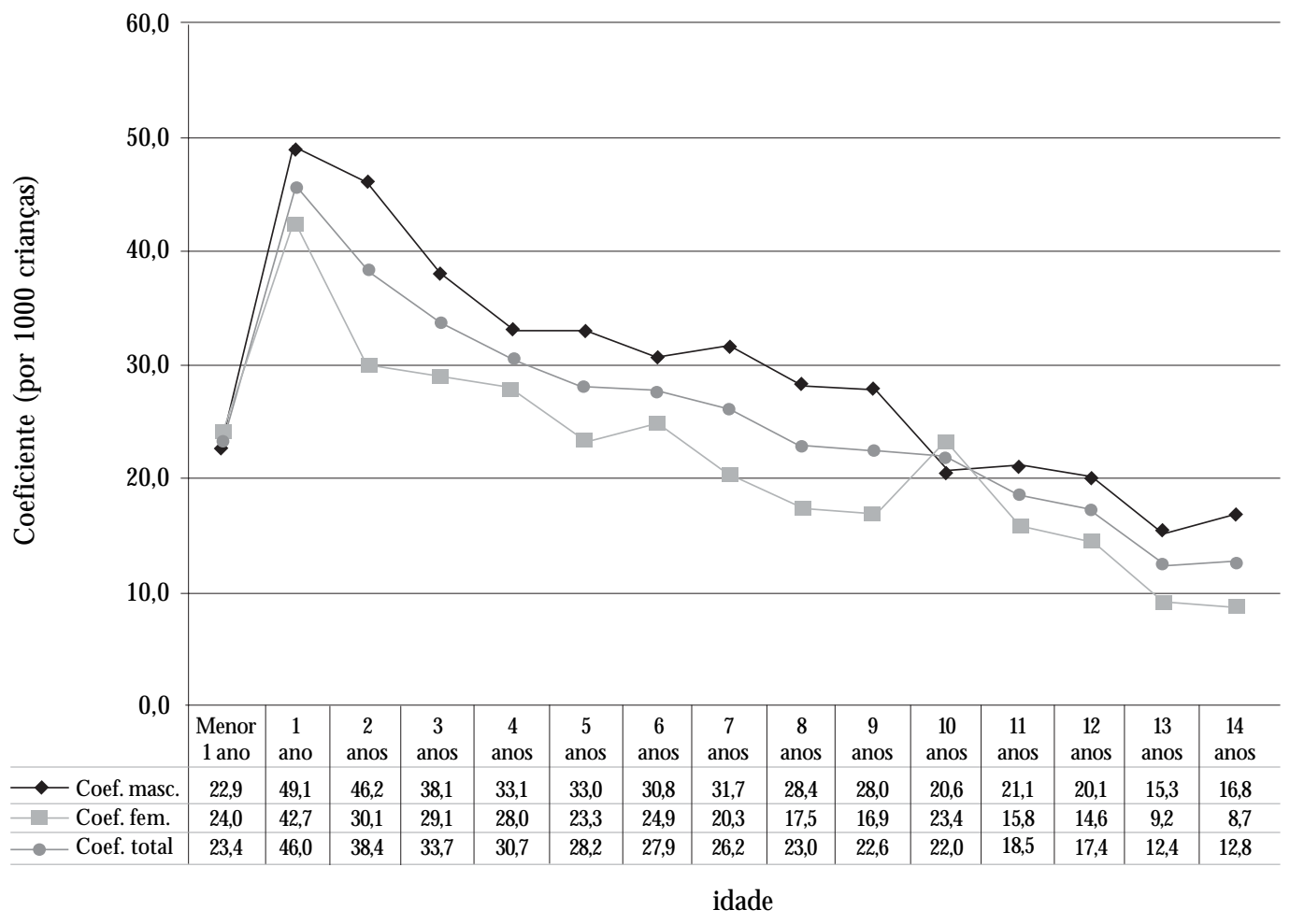

Gráfico 1. Coeficientes de Incidência de Quedas em menores de 15 anos segundo o sexo e a faixa etária, Londrina, 2001. 
Tabela 1. Distribuição dos menores de 15 anos vítimas de quedas segundo a região corpórea atingida, Londrina, 2001.

\begin{tabular}{lrr}
\hline \multicolumn{1}{c}{ Região corpórea atingida } & N & $\%$ \\
\hline Cabeça/pescoço & 1.650 & 55,2 \\
Ombro/membros superiores & 783 & 26,2 \\
Quadril/membros inferiores & 356 & 11,9 \\
Tórax/abdômen/dorso/coluna/pelve & 98 & 3,3 \\
Múltiplas regiões & 77 & 2,6 \\
Região não especificada & 27 & 0,9 \\
Total & 2.991 & $100,0^{*}$
\end{tabular}

*aproximado para 100,0\%.

Tabela 2. Distribuição dos menores de 15 anos vítimas de quedas segundo o tipo de lesão, Londrina, 2001.

\begin{tabular}{lrr}
\hline \multicolumn{1}{c}{ Tipo de lesão } & $\mathrm{N}$ & $\%$ \\
\hline Trauma superficial & 1.388 & 46,4 \\
Ferimento & 605 & 20,2 \\
Fratura & 361 & 12,1 \\
Luxação, entorse, distensão & 226 & 7,6 \\
Traumatismo de nervos & 6 & 0,2 \\
Traumatismo de vasos & 12 & 0,4 \\
Traumatismo intracraniano & 9 & 0,3 \\
Traumatismo aparelho urinário & 3 & 0,1 \\
e deórgãos pélvicos & & \\
Traumatismo não especificado & 381 & 12,7 \\
Total & 2.991 & 100,0 \\
\hline
\end{tabular}

pronto-socorro de uma criança de nove meses, por traumatismo intracraniano após queda de escada.

Os resultados encontrados permitem estimar que para cada óbito por queda ocorram 118 internações e 2.872 atendimentos de pronto-socorro.

\section{Discussão}

Quanto ao número de casos, dados semelhantes foram encontrados na investigação de Filócomo et al. ${ }^{9}$, na qual as quedas representaram $46,9 \%$ dos acidentes em menores de 15 anos. Baracat et al. ${ }^{5}$ também encontraram resultados similares, destacando a queda $(67,1 \%)$ como principal tipo de acidente na população pediátrica. As quedas $(44,9 \%)$ também são as principais causas de acidentes na infância segundo a pesquisa de $\mathrm{H}$ ara- da et al. ${ }^{12}$ e em um trabal ho realizado por Ballesteros et al. ${ }^{10}$, com crianças americanas menores de 15 anos, no qual as quedas foram responsáveis por $40 \%$ dos acidentes não fatais.

O predomínio do sexo masculino em relação às quedas é discutido por vários trabalhos ${ }^{10,18-20}$, concordando com os achados da presente investigação. 0 predomínio do sexo masculino está intimamente relacionado com o comportamento da família, aspectos sociais, educacionais eculturais. Aos meninos é concedida a liberdade de brincarem na rua sem a vigilância direta, além de as brincadeiras entre os meninos serem mais agressivas em relação às meninas. $\mathrm{A}$ associação desses fatores contribui para o maior número de acidentes entre as crianças do sexo masculino.

Em relação à faixa etária, resultados semeIhantes foram encontrados por Ballesteros et al. ${ }^{10}$, que em estudo com crianças americanas identificaram $29,0 \%$ das quedas na faixa etária de um a três anos, 25,6\% na faixa etária de 10 a 14 anos, $18,5 \%$ na faixa etária dequatro a seis anos, $15,8 \%$ dos sete aos nove anos e 5,2\% em menores de um ano. Resultados semelhantes foram encontrados por $\mathrm{M}_{\text {attos }}{ }^{21}$, que identificou as quedas como a principal causa de morbidade entre crianças de 0 a 12 anos. Já Filócomo et al. ${ }^{9}$ encontraram maior frequência das quedas em menores de um ano deidade (69,7\%) e $56,6 \%$ na faixa etária de um a três anos.

A frequência das quedas difere de acordo com a idade da criança, pois está diretamente relacionada com a etapa de desenvolvimento dela. Nos lactentes, os tipos de quedas mais frequentes são as quedas de colo, cama, sofá e carrinho de bebê, estando relacionadas em grande partecom o descuido do adulto responsável. Com o início do andar a partir de um ano de idade, aliado à sua curiosidade aguçada, a criança começa a explorar o meio e podem ocorrer as quedas da própria altura, devido à instabilidadee pouco equilíbrio, bem como as quedas de escadas, varandas ecadeirinhas de refeição, pois a criança jáécapaz de ficar em pé e começa a ter noção de espaço, sentindo atração pelos objetos que joga ao chão. A partir dos dois anos de idade, quando a criança adquire a habilidade de correr, as quedas da própria altura em razão de tropeção em objetos também ocorrem com bastante freqüência, assim como as quedas develocípede, motoca, equipamentos de playground e outros brinquedos. As quedas de janelas e em poços tornam-se uma preocupação importante nessa fase, na qual a criança é capaz de subir em móveis e al cançar as janelas e poços sem proteção. Já na fase escolar, 
são responsáveis pelas quedas as práticas de esporte, bem como bicicleta, patins, skate, entre outras atividades queenvolvem velocidade, além das quedas de árvore ${ }^{21}$.

A partir do conhecimento do desenvolvimento da criança associado ao tipo de acidente, aqui especificamente as quedas, é possível adotar medidas de segurança e vigilância eficazes. M aior vigilância dos lactentes, cuidado ao manuseá-los no colo, colocar proteção ao lado da cama ou sofá, carrinhos de bebê apropriados, áreas de lazer apropriadas com pisos emborrachados a fim de evitar o impacto das quedas, portão nas escadas para impedir o acesso da criança, proteção ao redor das varandas, piscinas, janelas e poços, uso de capacete e proteção durante as práticas esportivas podem contribuir para a manutenção da qualidade de vida e saúde de nossas crianças.

Em relação à região corpórea afetada, tal padrão vem sendo observado por estudos como 0 de Freitas et al. ${ }^{22}$, que identificou $41,2 \%$ de traumatismos cranioencefálicos decorrentes das quedas em crianças de um a dois anos de idade. $\mathrm{Ha}$ rada et al. ${ }^{12}$ também ressaltam a grande concentração detraumatismos cranioencefálicos em crianças vítimas de quedas. Segundo Baracat et al. ${ }^{5}$, as quedas da própria altura resultam em traumatismo cranioencefálico nas crianças menores de dois anos e em lesões de membros na população infantil maior de nove anos de idade. Em estudo realizado por Koizumi et al. ${ }^{13}$, as quedas foram as primeiras responsáveis por traumatismos cranioencefálicos em crianças menores de 10 anos.

0 traumatismo decabeça, principalmentena faixa etária de um a três anos, pode estar relacionado com a ausência de reflexos de proteção na criança pequena, o que não acontece na criança maior que já aprendeu a proteger a cabeça em situações de perigo, sendo então os membros mais atingidos. Tais fatos sugerem medidas de proteção já descritas anteriormente.

Foram poucos os estudos que relacionaram as quedas com o tipo de lesão decorrente desse tipo de acidente. Filócomo et al. ${ }^{9}$ observaram 0 ferimento como o terceiro tipo de lesão decorrentedeacidenteentremenores de 15 anos $(9,7 \%)$, e Lyons et al. ${ }^{20}$ identificaram um coeficiente alto de fraturas em crianças de 0 a 14 anos, mas não especificamente em relação às quedas.

Devido à prevalência de traumatismos superficiais, os achados nos permitem perceber a baixa gravidade das quedas. Todavia, os traumatismos intracranianos, apesar de sua baixa frequência, podem representar importante causa de mortalidade, uma vez que esse tipo de lesão foi responsável por um óbito em uma criança de nove meses de idade.

Em 2002, Filócomo et al. ${ }^{9}$ identificaram alguns agentes responsáveis pelas quedas em crianças, tais como cama (13,5\%), bicicleta ( $12,3 \%)$, escada $(9,2 \%)$, cadeira $(6,1 \%)$, muro $(6,1 \%)$ e outros. Harada et al. ${ }^{12}$ identificaram, entre as quedas, bicicleta, patins, escadas, berço, cadeira, cama, sofá, carrinho de bebê, muro e triciclo como os principais agentes desencadeadores de queda na população infantil.

Koizumi et al. ${ }^{13}$ identificaram, entre as crianças menores de 10 anos e internadas, que as quedas no mesmo nível de cama, cadeira e mobílias se destacaram entre os menores de um ano. Já na faixa etária de um a quatro anos, as quedas ocorreram de janela, escada, cama e cadeira. Ainda as quedas de janela, somadas às quedas de árvore e equipamentos de playground, foram mais frequentes na faixa etária de cinco a nove anos de idade.

Os equipamentos de playground também são destacados por Harada et al. ${ }^{23}$ como grande responsável pelas quedas, resultado da supervisão inadequada e, principalmente, do não cumprimento às normas de segurança da Associação Brasileira deN ormas Técnicas (ABNT), que prevê normas desde a instalação até a manutenção dos brinquedos. Portanto, o cuidado ea vigilância da criança em relação aos agentes aqui listados podem contribuir para a redução desses eventos.

Conhecer os agentes desencadeadores torna possível determinar ações de prevenção diretamente sobre eles. Podemos perceber, através dos agentes identificados, que as medidas de segurança podem ser adotadas facilmente pela família e responsáveis, evitando-se com isso internações eatendimentosdeurgênciae, principalmente, os óbitos decorrentes desse tipo de evento. Os achados sugerem que se orientem pais e responsáveis para que conheçam os riscos que acompanham as crianças nas suas diferentes fases de crescimento e desenvolvimento. Dessa forma, será possível adotar medidas de segurança, tendo como foco a eliminação dos agentes responsáveis por esses acidentes. U ma vez que anulemosa ação desses agentes, conseguiremos reduzir o índice dequedas na infância, proporcionando crescimento e desenvolvimento saudáveis.

Quanto ao nível de atendimento e evolução das vítimas, Koizumi et al. ${ }^{13}$ identificaram uma taxa de óbito por queda de 1,1\% em menores de 10 anos e $98,9 \%$ de sobrevida. Os achados da presente investigação demonstram a baixa gravidade em relação às quedas, porém a presença 
de óbito por essa causa leva à reflexão de que ele poderia ter sido evitado se medidas de segurança tivessem sido adotadas. As internações também poderiam ter sido evitadas, reduzindo-se inclusive os gastos hospitalares.

0 óbito de uma criança de nove meses de idade por queda da escada desperta para a questão da violência ou negligência, muitas vezes mascarada pelo relato de "acidentalidade" dos pais ou cuidador. Estudos apontam que a agressão corresponde a $72,0 \%$ dos traumas domésticos, cometidos principalmente pelo pai ou pela mãe ${ }^{24,25}$. A violência contra a criança, bem como a negligência, por suas repercussões físicas, emocionais e psicológicas, exige estudos aprofundados e contextualizados em razão da diversidade cultural, social e econômica que a envolve ${ }^{26}$.

\section{Conclusão}

0 fato deas quedas acontecerem, em grandeparte, envolvendo situações do ambiente doméstico sugere a sua modificação como forma efetiva de prevenção. Além dos cuidados com o ambiente doméstico, a supervisão direta sobre a criança parece ser a ação preventiva mais eficaz.
Para que 0 adulto responsável possa exercer os cuidados necessários na prevenção das quedas na infância, é preciso que el eadquira conhecimentos sobre os diferentes riscos nas diferentes fases de crescimento e desenvolvimento da criança. Para tanto, além da necessidade de intensificar ações de orientação e educação, individual ou coletiva, a atuação dos profissionais envolvidos nesse contexto precisa ser direcionada para cada etapa do desenvolvimento.

Diante da questão da violência e negligência embutidas nas causas aparentemente acidentais, os profissionais quelidam di reta eindiretamente com crianças necessitam estar alertas para a detecção dessas circunstâncias²7.

Destaca-se, ainda, a necessidade de estudos que venham a identificar e analisar fatores de risco para as quedas na população infantil, para que se possa atuar diretamente sobre as condições geradoras de tais acidentes. Ademais, em relação à grande proporção de atendimentos de pronto-socorro que não necessitaram de internação, torna-se importante habilitar os centros de atenção primária para os casos de baixa complexidade, a fim de diminuir a sobrecarga dos serviços terciários 5 .

\section{Colaboradores}

CBG Martins e SM Andrade participaram da concepção do trabalho, da análise e interpretação dos dados, de sua redação, da revisão crítica e da leitura e aprovação da versão final. 


\section{Referências}

1. Del Ciampo LA, Ricco RG. Acidentes na infância. Rev Pediatr 1996; 18:193-197.

2. Souza LJEX, Barroso M GT. Revisão bibliográfica sobre acidentes com crianças. Revista da Escola de Enfermagem da USP 1999; 33:107-112.

3. Souza LJEX, Barroso M GT. Acidente doméstico em crianças: abordagem conceitual. Acta Paulista de Enfermagem 1999; 70-77.

4. Krug, E. Injury: a leading cause of the global burden of disease. Geneva: World Health Organization; 1999. [acessado 2003 set 25]. Disponível em: www.who.int/violence_injury_prevention

5. Baracat ECE, Paraschin K, Nogueira RJN, Reis MC Fraga AM A, Sperotto G. Acidentes com crianças e sua evolução na região de Campinas, SP. J Pediatr 2000; 76:368-374.

6. Scherer EA, Sherer ZAP. A criança maltratada: uma revisão da literatura. Rev Latino Am Enfermagem 2000; 8:22-29.

7. Fonseca SS, Victora CG, Halpern R, Barros AJD, Lima RC, Monteiro LA, Barros F. Fatores de risco para injúrias acidentais em pré-escolares. J. Pediatr 2002; 78: 97-104.

8. Barros M DA, Ximenes R, Lima M LC. Mortalidade por causas externas em crianças e adolescentes: tendências de 1979 a 1995. Rev Saude Publica 2001; 35:142-149.

9. Filócomo FRF, Harada MJS, Silva CV, Pedreira M LG. Estudo dos acidentes na infância em um pronto-socorro pediátrico. Revista Latino Am Enf 2002; 10:41-47.

10. Ballesteros M F, Schieber RA, Gilchrist J, Holmgreen $P$, Annest JL. Differential ranking of causes of fatal versus non-fatal injuries among US children. Inj Prev 2003; 9:173-176.

11. Blank D. Conceitos básicos e aspectos preventivos gerais. In: Comitê de acidentes na infância. Manual de acidentes na infância e adolescência. São Paulo: Sociedade Brasileira de Pediatria; 1994. p. 2-13.

12. Harada MJCS, Botta M LG, Kobata CM, Szauter IH, Dutra G, Dias EC. Epidemiologia em crianças hospitalizadas por acidentes. Folha M ed 2000; 119:4347.

13. Koizumi MS, M ello Jorge MHP, Nóbrega LRB Waters $C$. Crianças internadas por traumatismo crânio-encefálico no Brasil, 1998: causas e prevenção. Inf Epidemiol SUS 2001; 10:93-101.

14. Hamley CA, Ward AB, Long J, Owen DW, Magnay $A R$. Prevalence of traumatic brain injury amongst children admitted to hospital in one health district: a population-based study. Accident Analysis and Prevention 2002; 33(1):44-50.

15. Organização Mundial de Saúde. Classificação Internacional de Doenças (CID-10). Centro Colaborador da OMS para a Classificação de Doenças em Português. $8^{a}$ ed. 10a revisão. São Paulo: EdUSP; 2000.
16. Dean AG, Dean JA, Coulombier D, Brendel KA, Smith DC, Burton AH, Dicker RC, Sullivan K, Fagan RF, Arner TG. EPI Info version 6: word processing, database and statistics program for epidemiology on microcomputers. Atlanta, USA: Center Disease Control and Prevention; 1995.

Instituto Brasileiro de Geografia e Estatística (IBGE).

17. Censo Demográfico 2000. M alha M unicipal Digital do Brasil. [acessado 2003 jun 26]. Disponível em: www.ibge.gov.br

Datasus. M ortalidade. [acessado 2003 jun 26]. Dis-

18. ponível em: www.datasus.gov.br/tabnet

Souza LJEX, Rodrigues AKC, Barroso M GT. A famí-

19. lia vivendo o acidente doméstico: relato de uma experiência. Rev Latino Am Enfermagem 2000; 8:8389.

Lyons RA, Delahunty AM, Kraus D, Heaven $M$,

20. Cabe M M, Allen H, Nash P. Children's fractures: a population based study. Inj Prev 1999; 5:129-132. $M$ attos IE. M orbidade por causas externas em crian-

21. ças de 0 a 12 anos: uma análise dos registros de atendimento de um hospital do Rio de Janeiro. Informe Epidemiológico do SUS 2001; 10(4):189-198. Freitas PEP, Oliveira QE, Nerung L, Henz DM , Ca-

22. mozzato A. Traumatismos crânio-encefálicos em crianças: estudo de 2.173 casos. Rev Assoc M éd Rio Grande do Sul (AM RIGS) 1999; 34:19-23.

Harada MJCS, Pedreira M LG, Andreotti JT. Segu-

23. rança com brinquedos de parques infantis: uma introdução ao problema. Rev Latino Am Enfermagem 2003; 11:383-386.

Pascolat G, Santos CFL, Eurico CRC, Valdez LCO,

24. Busato $D, M$ arinho $D H$. Abuso físico: o perfil do agressor e da criança vitimizada. J Pediatr 2001; $77(1): 35-40$

Lino Júnior W, Segal $A B$, Carvalho DE. Análise es-

25. tatística do trauma ortopédico infanto-juvenil no pronto-socorro de ortopedia de uma metrópole tropical. Acta Ortop Bras 2005; 13(4):179-182.

Ricas J, Donosa MTV, Greta M LM. A violência na

26. infância como uma questão cultural. Texto e Contexto Enfermagem 2006; 15(1):151-154.

Reichenheim ME, Dias AS, M oraes CL. Co-ocor-

27. rência de violência física conjugal e contra filhos em serviços de saúde. Rev Saude Publica 2006; 40(4):595-603.

Artigo apresentado em 30/04/2007

A provado em 25/06/2007

Versão final apresentada em 10/08/2007 